\title{
Association between periodontal disease and osteoporosis
}

\author{
Dan Piperea-Sianu', Adela M. Ceau², Mara Carsote ${ }^{3}$, Alexandru G. Croitoru ${ }^{4}$, \\ Stefan Cristea ${ }^{5}$ \\ 1Physiology Department, Faculty of Dental Medicine, \\ "Carol Davila" University of Medicine and Pharmacy, Bucharest, Romania \\ ${ }^{2}$ Politehnica University of Bucharest, Romania \\ ${ }^{3}$ Endocrinology Department, Faculty of General Medicine, \\ "Carol Davila" University of Medicine and Pharmacy, Bucharest, Romania \\ 4Physiopathology and Immunology Department, Faculty of Dental Medicine, \\ "Carol Davila" University of Medicine and Pharmacy, Bucharest, Romania \\ ${ }^{5}$ Orthopaedics and Traumatology Department, Faculty of General Medicine, \\ "Carol Davila" University of Medicine and Pharmacy, Bucharest, Romania
}

\begin{abstract}
Osteoporosis and periodontal disease (PD) are two chronic diseases, characterized by bone loss, with systemic or local impact (alveolar bone). Both pathologies have a progressive evolution, leading to systemic bone loss in the case of osteoporosis and bone lysis localized in the alveolar bone in the case of periodontal disease.

The present paper presents recent data from the literature on the association between periodontal disease and osteoporosis, on the role of cytokines in the bone resorption-apposition imbalance, and on how periodontal disease causes changes in serum levels of cytokines, leading to disorders in the systemic bone formation.

We also found it useful, especially for rheumatologists, to outline the extent to which periodontal disease can create a systemic context favorable to the development of osteoporosis.
\end{abstract}

Keywords: osteoporosis, periodontal disease, interleukins

\section{INTRODUCTION}

Osteoporosis is a condition characterized by decreased bone mass through demineralization, microarchitectural alterations of the bone tissue, resulting in increased bone fragility and high risk of fractures, even in the case of minor traumas. Osteoporosis represents a major public health issue through its impact on the quality of life (fear, movement limitation) but also through its social and economic impact (diagnosis costs, decreased work performance, treatment costs and also costs related to complications, including social assistance costs).

$\mathrm{PD}$ is a pathological complex that brings together the inflammatory or degenerative changes of the marginal periodontium.

The determining factor in the pathogenesis of PD is represented by microbial complexes with varying degrees of virulence, which progressively colonize the periodontal space, leading to bone lysis and loss of teeth by spontaneous avulsion.

Osteoporosis and PD are two chronic conditions that, through their increased incidence and prevalence, are of high interest to public health and clinical management.

Osteoporosis affects 200 million women worldwide $(1 / 10>60$ years, $1 / 5>70$ years, $2 / 5>80$ years, $1 / 3>90$ years), 75 million cases of osteoporosis being diagnosed in Europe, US and Japan. In Romania, $20 \%$ of the women over 55 years suffer from osteoporosis (1-3).

On a global level, PD affects between 10 and $15 \%$ of individuals, who suffer from moderate or severe periodontal impairment, whereas in Romania about $60 \%$ of individuals suffer from chronic periodontal impairment (4-6). 


\section{The relationship between periodontal disease and osteoporosis}

As osteoporosis is a disease characterized by decreased bone density, the hypothesis that it contributes to the loss of alveolar bone and, therefore, to periodontal impairment, can be raised. Thus, there has been a need for studies to assess the extent to which there is a uni- or bidirectional association between bone mineral density (BMD) and periodontal status.

Grocholewicz et al. (2012) have analyzed the relationship between BMD and the periodontal status. The results showed that bone density in the femoral neck correlates directly with the number of present teeth. Lumbar spine BMD correlates inversely with the severity of $\mathrm{PD}$, and radius BMD correlates inversely with the bleeding on probing index (BOP) (7).

In a study on 78 women in post-menopause, Singh A et al. (2014) concluded that the periodontal pockets depth (PPD), the clinical attachment loss (CAL), and the alveolar crest height are inversely correlated with the DXA T-score, the results being statistically significant. The ANOVA test showed statistically significant differences between the osteoporotic and osteopenic group, respectively, between the osteoporotic group and the periodontally healthy group, in terms of CAL, PPD and alveolar crest height (8).

Perreira FM et al. (2015) performed a 3-year longitudinal study on 33 patients divided into 3 groups, according to BMD. They were evaluated regarding periodontal health at the moment of the recruitment and, then, 3 years later. At the second visit, there was a statistically significant increase in BOP in the initially healthy patients, who had developed osteopenia or osteoporosis during the 3 year period. Also, the same significant increase was observed in the osteopenic patients who progressed to osteoporosis (9).

Mau LP et al. (2017) evaluated the risk of developing osteoporosis in patients with PD through a cohort study using data from Taiwan's national health base. They tracked 29,463 patients with PD, compared to 58,926 periodontally healthy individuals for a 6 year-long period, until death or until they were diagnosed with osteoporosis. The incidence of osteoporosis in the study group was $2.72 \%$ /year, compared to the control group - $1.66 \%$ / year. Also, patients with incipient, moderate and severe PD had a
$1.56,2.09$, or 2.08 higher risk of developing osteoporosis than the periodontally healthy ones (10).

Passos-Soares J.S. et al. (2017) have assessed the extent to which anti-osteoporotic treatment influences the severity of the periodontal impairment. The group $(\mathrm{n}=492)$ was divided into a study group $(n=113)$, who received anti-osteoporotic treatment at least during the previous 6 months, and a control group. The results showed that women who received anti-osteoporotic treatment with simple systemic estrogen or estrogen associated with progestin, calcium, and vitamin D had a lower degree of periodontal impairment, objectified by BOP, PPD and CAL. The prevalence of severe PD was $44 \%$ lower under treatment. Also, the DMFT index (decay, missing or filled teeth), which quantifies the tooth decay extent, averaged 20 in the treated group and 19 in the control group. The major component of the DMFT was represented by absent teeth (11).

Richa R.Y. et al. (2017) mention that the BOP, gingival and plaque indices were statistically significantly higher in osteoporotic patients compared to women with normal BMD. The CPITN periodontal index, following code distribution on sextants, showed that osteoporotic patients require more complex periodontal treatment compared to non-osteoporotic patients. Also, the association between periodontal impairment, BMD and menopause duration was statistically significant (12).

Savic Pavicin I. et al. (2017) mention the existence of an inverse correlation between the number of present teeth and the hip BMD and between the severity of periodontal impairment and BMD. Periodontally impaired patients, with at least one missing front tooth (incisor or canine), presented a lower BMD in comparison to individuals with no missing front tooth. The results were statistically significant (13).

After an extensive meta-analysis of the magnitude of the association between osteopenia, osteoporosis and PD, Brignardello-Petersen R. et al. (2017) conclude the following: (a) osteoporotic postmenopausal women, compared to non-osteoporotic and non-osteopenic women, showed a loss of epithelial insertion by $0,34 \mathrm{~mm}$ larger, $3.04 \%$ more periodontal pockets with $\mathrm{PPD} \geq 4 \mathrm{~mm}$ and $5.07 \%$ more periodontal pockets with $\mathrm{PPD} \geq 6 \mathrm{~mm}$; (b) compared to nonosteoporotic and non-osteopenic women, osteopenic postmenopausal women showed a $0.07 \mathrm{~mm}$ greater clinical attachment loss and $1.74 \%$ more periodontal pockets with $P P D \geq 4 \mathrm{~mm}$ (14). 


\section{The role of cytokines in the resorption-apposi- tion imbalance}

There are studies in the literature that link systemic inflammatory syndromes or localized inflammatory outbreaks to systemic or localized osteoporosis $(1,2)$.

A link between osteoporosis and inflammation also exists in terms of age, menopause and pregnancy. It is understandable how the mechanic, hormonal and nutritional factors influence these situations, but the relationship between osteoporosis and inflammation must be supported by evidence of a molecular nature, more specifically by cytokines as immunological mediators $(15,16)$.

The notions of ,immune-aging“ or ,immunosenescence" have been referred to in the literature to outline the increase of pro-inflammatory cytokine production with age. Franceschi $\mathrm{C}$ et al. and KiecoltGlaser JK mention the link between the biological senescence inflammatory syndrome and osteoporosis $(17,18)$.

The bone remodeling process requires a balance between resorption and apposition to maintain physiological limits of mineral density, bone mass, and quality of the process itself. Osteoporosis and PD are examples of disorders in which an imbalance occurs in the bone remodeling process. In the past, osteoporosis was regarded as a disease of the elderly. Currently, osteoporosis is known to be a multifactorial disorder that can occur at any age, involving metabolic, mechanical, nutritional and hormonal factors, resulting in compromised bone strength, the patient being prone to fractures (19-21).

The literature mentions the close relationship between systemic inflammatory diseases and osteoporosis. The main mechanism by which inflammation disturbs the balance between resorption and apposition is based on proinflammatory cytokines (Fig. 1). Activation of cellular and humoral immune response causes disorders regarding bone homeostasis, leading to osteoclastic stimulation, osteochondral destruction, and osteoblastic inhibition, resulting in the ending of bone formation $(19,20,22)$.

The increased production of proinflammatory cytokines - IL-1 $\beta$, IL-6, TNF- $\alpha$ - may be a common pathophysiological element for osteoporosis and PD. Systemic factors involved in the local tissue response to the infectious process related to PD may

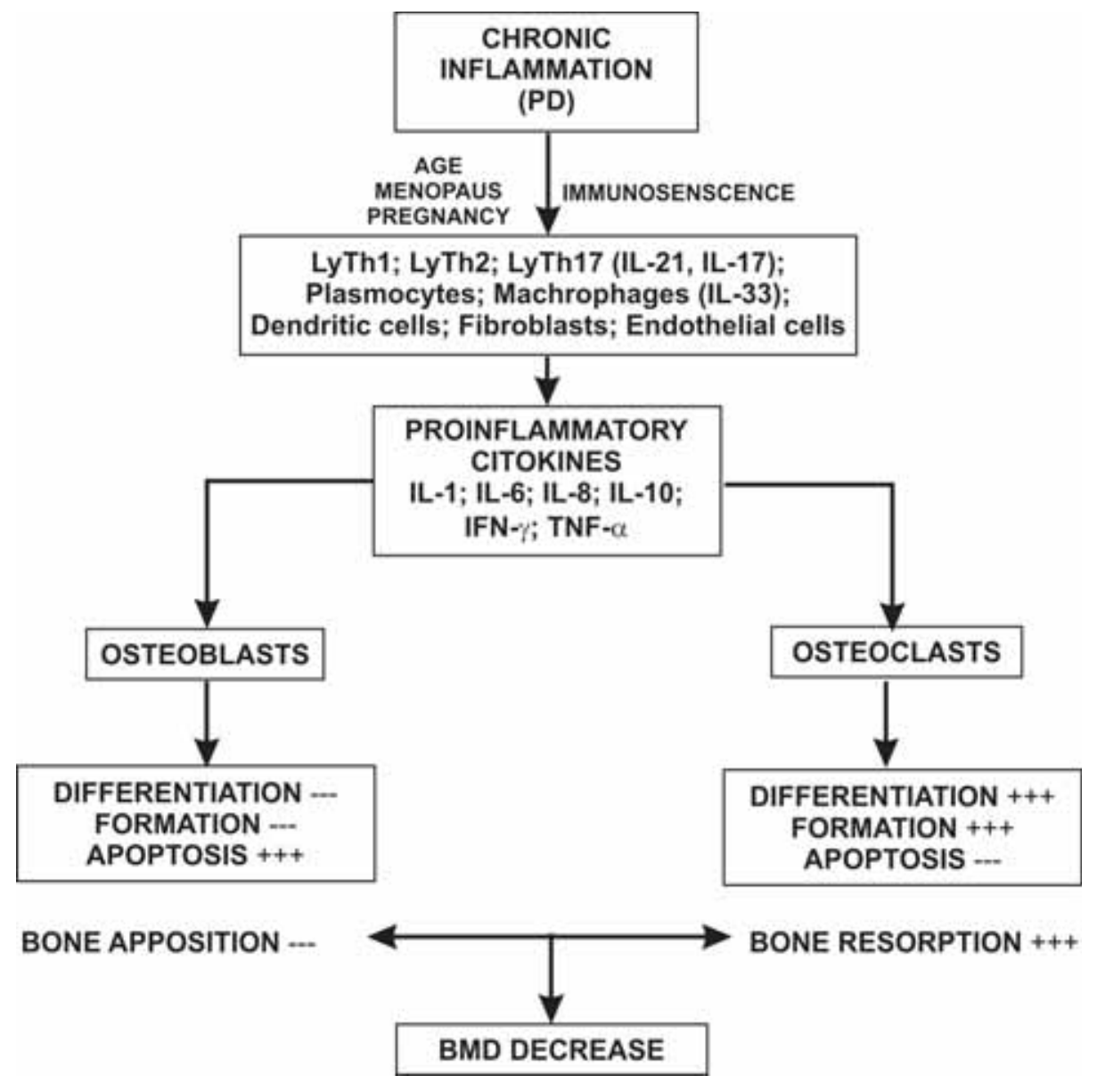

FIGURE 1. The involvement of proinflammatory cytokines in the disturbance of the bone resorption-apposition balance 
favor the bone remodeling imbalance, in favor of resorption. The link between proinflammatory cytokines and PD will be further detailed in the paper.

Interleukin-1 has a both direct and indirect osteolytic effect. The direct osteolytic effect is achieved through its action on osteoclasts, whereas the indirect effect is achieved by its ability to stimulate the synthesis of RANKL (the receptor activator of nuclear factor-kappaB ligand). Lee YM et al. mention the in-vitro osteolytic effect of IL-1. IL-1 also influences the formation of osteoclasts, 1.25-dihydroxycholecalciferol and RANK mediated, as well as the bone prostaglandin synthesis. The increase in prostaglandin levels may have a synergistic effect with IL1 , causing osteolysis, as prostaglandins are potent stimuli for bone resorption $(23,24)$.

Interleukin- 6 is a cytokine produced by osteoblasts or hematogenous medulla under the IL-1 or TNF- $\alpha$ stimulus. According to Lorenzo J, it has the following effects on the bone (23):

- influences the differentiation of osteoclast precursors;

- stimulates the formation of new osteoclasts independently from RANKL mechanism;

- can mediate the processes of senescence bone lysis;

- like IL-1, it can stimulate prostaglandin synthesis;

- stimulates the bone RANKL formation.

Interleukin- 8 is a chemokine produced by osteoclasts. Its osteolytic effect is given by the ability to stimulate the formation of new osteoclasts in a RANK-independent pathway, but also by the increase of NO synthase expression in osteoclasts $(23,25,26)$.

Interleukin-10 is a cytokine produced by activated lymphocytes. It has an inhibitory effect on the formation of osteoblasts and osteoclasts by altering the differentiation process of their precursors. Owens J et al. mention that IL-10 inhibits the formation of osteoclasts at the medullary level. At the periodontal level, Claudino $\mathrm{M}$ et al. observed in IL-10 deprived laboratory guinea pigs a smaller amount of alveolar bone and a decrease in osteoblastic differentiation indices. Wojdasiewicz P et al. assert that IL10 has a chondro-protective, anti-inflammatory action and lowers the synthesis of metallo-proteinases and nitric oxide (27-29).

The tumor necrosis factor- $\alpha$ (TNF- $\alpha$ ) is an inflammatory mediator, an acute phase reactant with certain osteoresorptive properties. According to Lorenzo J, it has the following effects $(23,30,31)$ :
- stimulates the formation of new osteoclasts;

- inhibits osteoclast apoptosis;

- increases calcemia by osteolysis;

- has an osteoclastogenic effect on osteoclastlike cell formation at a medullary level;

- inhibits new bone formation and osteoblast function in general;

- stimulates osteoblast apoptosis;

- inhibits the production of insulin-like growth factor (IGF).

Interferon- $\gamma(\mathrm{IFN}-\gamma)$ is a cytokine produced by $\mathrm{T}$ lymphocytes, with an important role in inflammation, having a wide variety of biological effects. Its role in the resorption-apposition balance is not entirely elucidated at present. It seems that depending on the biological context, IFN- $\gamma$ can cause disruption in both directions. It is mentioned that in vitro it can have an inhibitory effect of osteolysis, on the one hand, by the ability to disrupt the differentiation of osteoclast precursors and, on the other hand, by blocking 1,25-dihydroxycholecalciferol and IL-1 from forming osteoclast-like cells at a medullary level. The osteoresorptive effect of IFN- $\gamma$ was observed in vitro, through its the capacity of osteoblastic inhibition and stimulation of $\mathrm{T}$ lymphocytes to produce TNF- $\alpha$ and RANKL. In vivo, the osteoresorptive effect is reported by Mann GN et al., who attained osteopenia in laboratory guinea pigs which were administered IFN- $\gamma$, intraperitoneally, for one week. In a recent study on 3 types of guinea pigs, Gao Y et al. attempted to demonstrate that the osteoresorptive effect of IFN- $\gamma$ is a resultant of its direct and indirect effects. They note that the role of IFN- $\gamma$ in osteoclastogenesis is based on stimulating the $\mathrm{T}$ lymphocyte activation. They also conclude that under conditions of estrogen deficiency and infection, the overall effect of IFN- $\gamma$ is represented by shifting the resorption-apposition balance to the left, resulting in demineralization and bone loss $(23,32,33)$.

\section{Influence of periodontal health on BMD}

The common pathophysiological element between PD and osteoporosis is represented by bone loss. PD involves the damage of the superficial component of the marginal periodontium (destruction of the gingiva and supra-alveolar ligaments) and also of the profound component of the marginal periodontium (loss of alveolar bone). Osteoporosis is a skeletal pathology that involves lowering bone strength by affecting bone quality and bone density. 
Due to the similarities between the two conditions, the results of the progression of the two may be similar. Also, the two conditions have common risk factors such as age, estrogen deficiency and aspects related to the individual's lifestyle. People with lower income and underprivileged education tend to have unhealthy habits, such as smoking, alcohol abuse and over-consumption of unhealthy insubstantial meals. These may favor the development of both PD and osteoporosis, by starting and amplifying the inflammatory syndrome.

The mechanism that lies behind the accentuated bone resorption consists of increased systemic osteoclastic activity, namely local cell activity and influence of proinflammatory cytokines. Both systemic and local inflammatory syndromes, such as PD, are characterized by a degree of bone resorption.

In the physiological bone remodeling process, intercellular contact between osteoblasts and osteoclastic monocytes/precursors expressing RANKL is essential. In inflammation, activated $\mathrm{T}$ lymphocytes can express RANKL, so intercellular contact between activated $\mathrm{T}$ lymphocytes and osteoclastic monocytes/precursors expressing RANKL may be involved in osteoclastogenesis. Also in inflammation, B lymphocytes can express RANKL by participating in the osteoclast formation (34).

Inflammatory infiltrates, consisting of osteoclast monocytes/precursors and $\mathrm{T}$ lymphocytes, in the superficial and profound components of the marginal periodontium, may occur in PD. As mentioned earlier, intercellular contact between these two cell categories expressing RANKL is responsible for osteoclast formation in PD. Liu D et al. also mention that patients with advanced PD have an increased RANKL expression and a decreased expression of OPG (osteoprotegerine - role in inhibiting the activation of mature osteoclasts) $(34,35)$.

A common risk factor between osteoporosis and PD is the deficiency of estrogenic hormones that causes bone metabolic imbalance, resulting in decreased BMD. This phenomenon occurs by increasing the number of osteoclasts in estrogen-deficient conditions, as a result of proinflammatory cytokine action. Decreased serum levels of estrogen hormones directly or indirectly cause changes in the levels of proinflammatory cytokines IL-1 and TNF- $\alpha$ that are involved in osteoclastogenesis and inhibition of osteoblast formation. Also, PD determines a local inflammatory process with systemic repercussions. Thus, there is a local increase in the concen- trations of proinflammatory cytokines and prostanoids, leading to bone resorption by activation of osteoclasts (23).

The important element in the PD-osteoporosis relationship is the extent to which periodontal damage overcomes the local alveolar barrier, influencing the systemic BMD.

\section{Interleukin-1}

We have been able to find in the literature four studies that mention the increase in serum IL-1 in periodontal impairment. Górska $\mathrm{R}$ et al., in a study on 25 patients with severe periodontal impairment (at least one periodontal pocket with PPD $>5 \mathrm{~mm}$ ) and 25 periodontally healthy subjects, have reported a direct correlation between PD and serum and gingival IL-1 . Orozco A et al. reported detectable serum levels of IL-1 in periodontally impaired patients. Zahraa $\mathrm{F}$ et al., in a study on $50 \mathrm{PD}$ patients aged between 23 and 60 years compared with 25 periodontally healthy individuals, reported a higher serum IL-1 concentration in patients with chronic $\mathrm{PD}$, the results being statistically significant (IL-1 $\beta$ : control group $27.21 \mathrm{pg} / \mathrm{mL}$, PD $34.57 \mathrm{pg} / \mathrm{mL}$ ). They also mention a correlation between serum IL-1 and PD indices such as plaque index, gingival index, PPD and CAL. Stefanovska E et al. reported statistically significantly higher serum concentrations of IL-1 in patients with gingivitis and periodontitis $(n=60)$ in comparison to the control group $(n=30)$, as follows: IL-1 $\alpha / \mathrm{IL}-1 \beta$ : control group $1.82 / 0.09 \mathrm{pg} /$ $\mathrm{mL}$, gingivitis 2.13/0.35 $\mathrm{pg} / \mathrm{mL}$, periodontitis 2.46/0.31 pg/mL (36-39).

\section{Interleukin-6}

Recent data from the literature show a correlation between serum IL-6 and periodontal impairment. Reddy B, in a study on 111 periodontally impaired patients (77 with localized aggressive periodontitis, 34 with generalized aggressive periodontitis), have investigated the correlation between the severity of periodontal damage and serum IL-6 concentration. The results showed that the average IL- 6 serum concentration was higher in patients with local aggressive periodontitis compared to the control group (3.53 pg/mL \pm 2.015 and $2.28 \mathrm{pg} / \mathrm{mL} \pm 1.07$, respectively). Gani DK et al. investigated serum IL-6 on 42 individuals divided into control group $(\mathrm{n}=14)$, localized PD $(n=14)$ and generalized PD $(n=14)$. The median of the serum concentrations of IL- 6 was 6.35 $\mathrm{pg} / \mathrm{mL}$ in the generalized periodontal impairment group and below $5 \mathrm{pg} / \mathrm{mL}$ in the case of localized 
periodontal impairment, respectively the control group. Loose BG et al. reported higher serum levels of IL- 6 in periodontally impaired patients in their group ( $\mathrm{n}=107)$, versus control. Also, $52 \%$ of patients with generalized PD, $36 \%$ of those with localized periodontal impairment, and only $26 \%$ of the control group, were IL-6 seropositive. Robati M et al. reported higher serum IL-6 concentration in patients with aggressive PD compared to the control group (19.07 $\mathrm{pg} / \mathrm{mL} \pm 2.11$ and $1.81 \mathrm{pg} / \mathrm{mL} \pm 0.51$, respectively). There is a strong direct association between PD and IL-6 as a proinflammatory cytokine (40-43).

\section{Interleukin-8}

In a recent study on 88 patients divided into 4 groups (control group, incipient PD, moderate PD, advanced PD), Tâlvan E.T. et al. analyzed the serum concentrations of several cytokines in correlation with the degree of periodontal damage. The results showed that IL- 8 and IL-1 $\beta$ had higher serum concentrations in patients with periodontal damage, regardless of group, compared to the control group, the results being statistically significant. Also, a strong statistically significant correlation was found between IL- $1 \beta$ and IL- 8 in patients with moderate PD, meaning that serum IL-8 varies proportionally to IL$1 \beta$ in these patients. In terms of advanced PD, the same direct correlation between IL- 8 and IL- $1 \beta$ is mentioned, but also an inverse correlation between IL-8 and IL-10, meaning that serum IL-8 increases proportionally to the decrease in serum IL-10 concentration in these patients. Another study of AlRassam Z.T. et al. on an Iraqi population reported higher IL-8 serum concentration in periodontally impaired patients $(\mathrm{n}=81) \quad(59,195 \mathrm{pg} / \mathrm{mL} \pm 26,56)$ versus control $(\mathrm{n}=18)(25.05 \mathrm{pg} / \mathrm{mL} \pm 6.109)$, the results not being statistically significant $(44,45)$.

\section{Interleukin-10}

Interleukin-10 is a cytokine having an anti-inflammatory role in regulating the immune response in PD. It has beneficial effects through inhibiting tissue destruction, proinflammatory cytokine synthesis, and immune response in general. Tâlvan E.T. et al. mention a decrease in serum IL-10 with age (3130 years: $36.80 \mathrm{pg} / \mathrm{mL}, 61-70$ years: $15.69 \mathrm{pg} / \mathrm{mL}$ ), suggesting a decrease in the protective effect consecutive to senescence. Also, the serum concentration of IL-10 was reported to be significantly greater in the control group, comprised of periodontally healthy individuals, being correlated inversely with the degree of periodontal impairment (control 34.21 $\mathrm{pg} / \mathrm{mL}$, incipient PD $28.2 \mathrm{pg} / \mathrm{mL}$, moderate PD
$15.38 \mathrm{pg} / \mathrm{mL}$, advanced PD $11.33 \mathrm{pg} / \mathrm{mL}$ ). Also in advanced PD, serum IL-10 varies inversely proportional to IL-8, the results being statistically significant (44).

Passoja A. et al. have investigated the systemic impact of PD on the variation of IL-10 as an antiinflammatory cytokine and on the variation of TNF- $\alpha$ as a pro-inflammatory cytokine. The study group consisted of 61 patients with PD and the control group of 30 periodontally healthy individuals. They reported: (a) the mean serum concentration of IL-10 was higher in the control group, whereas the mean serum concentration of TNF- $\alpha$ was lower, compared to the periodontally impaired patients; (b) the serum TNF- $\alpha / \mathrm{IL}-10$ ratio was about three times higher in PD, regardless of the degree of periodontal impairment, compared to the control group; (c) serum IL-10 correlated inversely with the periodontal impairment indices (BOP, PPD, CAL); (d) serum TNF- $\alpha$ correlated directly with the degree of periodontal impairment, the results being statistically significant only between moderate and severe impairment. (45)

\section{TNF- $\alpha$ and IFN- $\gamma$}

Andrukhov O. et al. have investigated the variation in serum cytokine concentrations in relation to the oral microbial load found in the subgingival dental plaque, determined by direct semiquantitative PCR. They reported higher serum concentrations of TNF- $\alpha$ and IFN- $\gamma$ in PD compared to controls. Serum concentrations of TNF- $\alpha$ and IFN- $\gamma$ also correlated directly with the amount of periodontal pathogens Aggregatibacter actinomycetemcomitans and Porphyromonas gingivalis. Zeyad T et al. also mention higher levels of TNF- $\alpha$ and IFN- $\gamma$ in PD (453.9 $\mathrm{ng} / \mathrm{L} \pm 397.1$ and $333.1 \mathrm{ng} / \mathrm{L} \pm 279.3)$ in comparison to healthy individuals $(300,6 \mathrm{ng} / \mathrm{L} \pm 16.4$ and $280.8 \mathrm{ng} /$ $\mathrm{L} \pm 20.14$ ), but the results are not statistically significant. With regard to the impact of periodontal therapy, Koppolu P et al. performed a study on 40 PD patients, who were divided into two groups. Group A did not receive periodontal therapy, and group B received non-surgical periodontal therapy consisting of subgingival scaling and root planing. After 8 weeks, they reported a decrease in serum TNF- $\alpha$ values as follows: group A (initially $22.85 \pm 1.29 \mathrm{pg} / \mathrm{dl}$, after 8 weeks $22.68 \pm 1.23 \mathrm{pg} / \mathrm{dl}$ ), group B (initially $22.14 \pm 1.46 \mathrm{pg} / \mathrm{dl}, 8$ weeks post-treatment $20.20 \pm$ $1.61 \mathrm{pg} / \mathrm{dl}$ ). The results are statistically significant for group B. Duarte PM et al. evaluated the serum concentrations of TNF- $\alpha$ and IFN- $\gamma$ in 28 patients 
with chronic PD and aggressive PD and 14 periodontally healthy individuals, as well as the periodontally impaired patients' evolution under treatment. The results showed higher concentrations of pretreatment proinflammatory cytokines in aggressive PD. 6 months after the periodontal treatment, a statistically significant decrease in TNF- $\alpha$ was observed in the aggressive PD group. Gorrska R. et al. reported higher serum concentrations of TNF- $\alpha$ and IFN- $\gamma$ in PD compared to healthy patients, the results being statistically significant. Zong $\mathrm{M}$ et al. also mention a higher concentration of serum IFN- $\gamma$ in PD patients from his study, compared to the control group, and a decrease in IFN- $\gamma$ after initial periodontal treatment $(36,45,47-50)$.

\section{CONCLUSIONS}

Recent data from the literature show an association between PD and osteoporosis. They mention that osteoporotic patients have a higher degree of periodontal damage compared to healthy individuals. Periodontal impairment is quantified by plaque index, bleeding on probing, number and depth of periodontal pockets, clinical attachment loss, and number of present teeth. We mention that older studies reported contradictory results in this association. Recently, with the improvement of diagnostic meth- ods, we noticed a tendency towards uniformity in the sense that a consensus seems to be shaping on the association of PD-osteoporosis.

Moderate and severe PD involves the deterioration of the profound component of the marginal periodontium. This results in a local inflammatory response, which, as we have seen, occurs at the systemic level by increasing the concentration of proinflammatory cytokines and decreasing the concentration of anti-inflammatory cytokines. These cytokines can influence the bone resorption-apposition balance, decreasing the systemic bone mineral density. Thus, seemingly insignificant, a local inflammatory process in the alveolar bone generated by $\mathrm{PD}$, through systemic consequences, may favor the installation of systemic osteoporosis.

The non-surgical treatment of PD has been shown to reduce serum levels of proinflammatory cytokines, in addition to relieving local suffering. Thus, we consider useful for rheumatologists to acknowledge the need for interdisciplinary collaboration in these patients. Local non-surgical periodontal treatment (subgingival scaling and root planing), associated with the main anti-osteoporotic treatment, may have positive consequences for the overall therapeutic success in these patients.

Conflict of interest: none declared Financial support: none declared

\section{REFERENCES}

1. Kanis J.A., on behalf of the World Health Organization Scientific Group Assessment of osteoporosis at the primary healthcare level. Technical report University of Sheffield, UK: WHO Collaborating Centre; 2008

2. EFFO and NOF, Who are candidates for prevention and treatment for osteoporosis?. Osteoporos Int. 1997;7(1):1-6.

3. Grigorie D., Sucaliuc A., Ivan M. et al. High prevalence of vitamin D deficiency in 1048 Romanian women with postmenopausal osteoporosis. Acta Endocrinologica, 2008; 4(1): 33-45.

4. Croitoru G.Al., Mihai C., Piperea-Şianu D. et al. Implicaţiile infecției parodontate la pacienţii cu poliartrită reumatotdă, RoJRheumatol, 2013; 22:69-73.

5. Papapanou P.N. Periodontal diseases: epidemiology, Ann Periodontol, 1996; 1:1-36.

6. Păduraru A., Vataman R., Sălceanu M. et al. Epidemiological study regarding prevalence, distribution and severity of periodontal disorders in a study group aged between 15-65 years, Rev Med Chir Soc Med Nat lasi, 2010; 114(4):1178-83.

7. Grocholewicz K., Bohatyrewicz A. Oral health and bone mineral density in postmenopausal women. Arch Oral Biol. 2012 Mar; 57(3):245-51.

8. Singh A., Sharma R.K., Siwach R.C. et al. Association of bone mineral density with periodontal status in postmenopausal women. J Investig Clin Dent. 2014 Nov;5(4):275-82

9. Pereira F.M., Rodrigues V.P., de Oliveira A.E. et al. Association between periodontal changes and osteoporosis in postmenopausal women. Climacteric. 2015 Apr;18(2):311-5
10. Mau L.P., Kuan Y.C., Tsai Y.C. et al. Patients with chronic periodontitis present increased risk for osteoporosis: A populationbased cohort study in Taiwan. J Periodontal Res. 2017 May 2. doi: 10.1111/jre.12464

11. Passos-Soares J.S., Vianna M.I.P., Gomes-Filho I.S. et al. Association between osteoporosis treatment and severe 24(7):789-795

12. Richa R.Y., Puranik M.P., Shrivastava A. Association between osteoporosis and periodontal disease among postmenopausal Indian women. J Invest Clin Dent. 2017;8(3). doi: 10.1111/ jicd. 12223

13. Savić Pavičin I., Dumančić J., Jukić T. et al. The relationship between periodontal disease, tooth loss and decreased skeletal bone mineral density in ageing women. Gerodontology. 2017; $00: 1-5$.

14. Brignardello-Petersen $\mathbf{R}$. The magnitude of an association between periodontal attachment loss and osteoporosis or osteopenia is small. J Am Dent Assoc. 2017;148(4):e41.

15. Ginaldi L., Di Benedetto M.C., De Martinis M. Osteoporosis, inflammation and ageing. Immun Ageing. 2005; 2:14.

16. Yun A.J., Lee P.Y. Maldaptation of the link between inflammation and bone turnover may be a key determinant of osteoporosis. Med Hypotheses. 2004; 63(3):532-7

17. Franceschi C., Bonafè M., Valensin S. Inflamm-aging. An evolutionary perspective on immunosenescence. Ann N Y Acad Sci. 2000 Jun; 908:244-54 periodontitis in postmenopausal women. Menopause. $2017 \mathrm{Jul}$; 
18. Kiecolt-Glaser J.K., Preacher K.J., MacCallum R.C. Chronic stress and age-related increases in the proinflammatory cytokine IL-6. Proc Natl Acad Sci U S A. 2003 Jul 22;100(15):9090-55.

19. Amarasekara D.S., Yu J., Rho J. Bone Loss Triggered by the Cytokine Network in Inflammatory Autoimmune Diseases. J Immunol Res. 2015;2015:832127

20. Feng X., McDonald J.M. Disorders of bone remodeling. Annu Rev Pathol. 2011;6:121-45

21. Takayanagi H. Osteoimmunology and the effects of the immune system on bone. Nat Rev Rheumatol. 2009 Dec;5(12):667-76

22. Redlich K., Smolen J.S. Inflammatory bone loss: pathogenesis and therapeutic intervention. Nat Rev Drug Discov. 2012 Mar 1; 11(3):234-50

23. Marcus R., Feldman D., Dempste D. et al. Osteoporosis. 4th Edition. Academic Press. 2013, eBook ISBN: 9780123982520

24. Lee Y.M., Fujikado N., Manaka H. et al. IL-1 plays an important role in the bone metabolism under physiological conditions. Int Immunol. 2010 Oct; 22(10):805-16

25. Kim M.S., Day C.J., Morrison N.A. MCP-1 is induced by RANKL, promotes osteoclast fusion and rescues GM-CSF suppression of osteoclast formation. J Biol Chem. 2005;280(16):16163-9.

26. Bendre M.S., Margulies A.G., Walser B. et al. Tumor-derived interleukin-8 stimulates osteolysis independent of the receptor activator of nuclear factor-kappaB ligand pathway. Cancer Res. 2005 Dec 1;65(23):11001-9

27. Owens J., Chambers T.J. Differential regulation of osteoclast formation: interleukin 10 (cytokine synthesis inhibitory factor) suppresses formation of osteoclasts but not macrophages in murine bone marrow cultures. J Bone Miner Res. 1995; 10(Suppl.1):S220.

28. Claudino M., Garlet T.P., Cardoso C.R. et al. Down-regulation of expression of osteoblast and osteocyte markers in periodontal tissues associated with the spontaneous alveolar bone loss of interleukin-10 knockout mice. Eur J Oral Sci. 2010;118(1):19-28.

29. Wojdasiewicz P., Poniatowski Ł.A., Szukiewicz D. The Role of Inflammatory and Anti-Inflammatory Cytokines in the Pathogenesis of Osteoarthritis. Mediators of Inflammation. 2014; (2014):561459.

30. Kitaura H., Kimura K., Ishida M. et al. Immunological Reaction in TNF-a-Mediated Osteoclast Formation and Bone Resorption In Vitro and In Vivo. Clinical and Developmental Immunology. 2013; (2013):181849.

31. Mollazadeh S., Fazly Bazzaz B.S., Kerachian M.A. Role of apoptosis in pathogenesis and treatment of bone-related diseases. Journal of Orthopaedic Surgery and Research. 2015;10:15.

32. Mann G.N., Jacobs T.W., Buchinsky F.J. et al. Interferongamma causes loss of bone volume in vivo and fails to ameliorate cyclosporin A-induced osteopenia. Endocrinology. 1994; 135:1077-83.

33. Gao Y., Grassi F., Ryan M.R. et al. IFN-gamma stimulates osteoclast formation and bone loss in vivo via antigen-driven $\mathrm{T}$ cell activation. J Clin Invest. 2007;117(1):122-32.

34. Kim J., Amar S. et al. Periodontal disease and systemic conditions: a bidirectional relationship. Odontology. 2006; 94(1):10-21

35. Liu D., Xu J.K., Figliomeni L., Huang L. et al. Expression of RANKL and OPG mRNA in periodontal disease: possible involvement in bone destruction. Int J Mol Med. 2003 Jan; 11(1):17-21.

36. Górska R., Gregorek H., Kowalski J. et al. Relationship between clinical parameters and cytokine profiles in inflamed gingival tissue and serum samples from patients with chronic periodontitis. J Clin Periodontol. 2003;30(12):1046-52

37. Orozco A., Gemmell E., Bickel M. et al. Interleukin-1beta, interleukin-12 and interleukin-18 levels in gingival fluid and serum of patients with gingivitis and periodontitis. Oral Microbiol Immunol. 2006; 21(4):256-60.

38. Shake Z., Hashem B. Study the role of proinflammatory and anti- inflammatory cytokines in Iraqi chronic periodontitis patients. Journal Of Baghdad College Of Dentistry. 2016; (24):164-9.

39. Stefanovska E., Nakova M., Ivanovski K. et al. Interleukin-1 (ILI- $\alpha$ and ILI- $\beta$ ) in gingival fluid and serum of patients with gingivitis and periodontitis. Balkan Journal of Stomatology. 2012;16(1):34-8.

40. Reddy B. Interleukin-6 Levels in Generalized and Localized Aggressive Periodontitis Patients. Virginia Commonwealth Univeristy Richmond, Virginia June, 2004.

41. Gani D.K., Lakshmi D., Krishnan R. et al. Evaluation of C-reactive protein and interleukin- 6 in the peripheral blood of patients with chronic periodontitis. Journal of Indian Society of Periodontology. 2009; 13(2):69-74

42. Loos B.G., Craandijk J., Hoek F.J. et al. Elevation of systemic markers related to cardiovascular diseases in the peripheral blood of periodontitis patients. J Periodontol. 2000;71(10):1528-34.

43. Robati M., Ranjbari A., Ghafourian Boroujerdnia M. et al. Detection of IL-4, IL-6 and IL-12 serum levels in generalized aggressive periodontitis. Iran J Immunol. 2011;8(3):170-5.

44. Tâlvan E.T., Mohor C., Chisnoiu D. et al. Expression of Interleukin (IL)-1 $\beta$, IL-8, IL-10 and IL-13 in Chronic Adult Periodontitis Progression. Arch Med. 2017; (9):1-8.

45. Al-Rassam Z., Taha M. Serum Cytokines Profiles and Some Salivary Parameters in Chronic Periodontitis Patients in Mosul - Iraq. International Journal Of Sciences: Basic And Applied Research (IJSBAR). 2014;16(1):339-350.

46. Passoja A., Puijola I., Knuuttila M. et al. Serum levels of interleukin-10 and tumour necrosis factor- $\alpha$ in chronic periodontitis. J Clin Periodontol. 2010 Oct;37(10):881-7

47. Andrukhov O., UIm C., Reischl H. et al. Serum cytokine levels in periodontitis patients in relation to the bacterial load. Periodontol. 2011 Jun;82(6):885-92

48. Koppolu P., Durvasula S., Palaparthy R. et al. Estimate of CRP and TNF-alpha level before and after periodontal therapy in cardiovascular disease patients. Pan Afr Med J. 2013; 15: 92

49. Duarte P.M., da Rocha M., Sampaio E. et al. Serum levels of cytokines in subjects with generalized chronic and aggressive periodontitis before and after non-surgical periodontal therapy: a pilot study. J Periodontol. 2010 Jul;81(7):1056-63.

50. Zong M., Yang P.S., Qi X.M. et al. Changes of circulating IFNgamma, IL-4 in patients with chronic periodontitis before and after periodontal initial therapy. Shanghai Kou Qiang Yi Xue. 2005 Apr;14(2):131-3. 